Sis

FED O :
SLAC-PEP-NOTE--269

DE89 006238

\title{
Damping Coherent Phase Dscillations by Means of Path-Length Modulation
}

John R. Rees

\section{Introduction}

iulti-bunch storage rings and synchrotrons are typically piagued by a tendency for the bunches to indulge in unstable coherent phase oscilTations engendered by their electromagnetic interactions with the vaclium chamber. In many machines feedback systems have been used successfully to damp these oscillations using a signal proportional to the coherent phase motion or the concomittant energy motion to control an auxilliary longitudinat electric field. The purpose of this note is to describe an alternative feedback systen which, using the same kind of a signal, modulates the path length of the orbit of the reference particie (the synchronous particle in the absence of coherent phase oscillations) in such a way as to damp coherent oscillations.

The Method

For the purpose of 11 lustrating the method, we may direct our attention to a storage ring containing only a single bunch and stipulate that the path-length modulation is achieved by a controlled beam bump produced by two fast magnets, or kickers, located exactly one-half radial betatron wavelength apart. These kickers are powered so as to produce a radial orbit distortion in the half-wavelength region between them and none whatever outside that region. See Fig. 1. The amplitude of this bump may be varied from one passage of the bunch to the next in response to the feedback signal. We further stipulate that some of the ring bending magnets lie within the beam bump so that the net path length for the iference particle through the bump varies with the amplitude of the bump in first order. Let us call the increase of net path length due solely

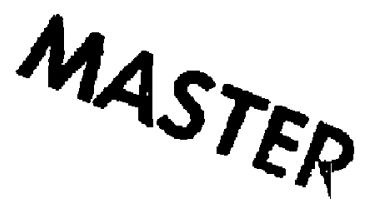


to the controlled bump is remembering that it is a variable dependent on a feedback signar.

In the development to follow we shall use the notation of Sands. Equation (3.32) states that for any particle in an ordinary electron storage ring

$$
\frac{d \underline{I}}{d t}=-\alpha \frac{\underline{E}}{E_{0}} .
$$

Summing over all particles in the bunch and prohibiting "internal" coherent phase motion (with which this kind of feedback system is not designed to cope) we find that the same equation applies if $\underline{I}$ and $\underline{\varepsilon}$ are interpreted as the mean time and energy displacenent, respectively, of the bunch due to coherent motion. Henceforward, we shall so interpret these symbols. With the introduction of the controlled beam bump the equation is modified to read

$$
\frac{d \underline{I}}{d t}=-a \frac{\underline{E}}{E_{0}}-\frac{\delta s}{L} .
$$

Confining ourselves to small-amplitude oscillations since we expect to damp them anyway, we adopt Eq. $(3.40)$ from Sonds neglecting the radiation term which would only lead to enhanced damping

$$
\frac{d \underline{E}}{d t}=\frac{e \dot{V}}{T_{0}} \underline{I} \text {. }
$$

Combining this equation with Eq.(1) above we get

$$
\frac{d^{2} I}{d t^{2}}+\frac{d}{d t}\left(\frac{\delta s}{L}\right)+\Omega^{2} \underline{I}=0
$$


which tells us immediately that is must be made proportional to $\underline{I}$ by the feedback signal if it is to effect damping. If it were made proportional to $\underline{\varepsilon}$ it would shift the frequency of coherent oscillation but not enhance the damping. The coherent dariping rate of due to the feedback system is

$$
a_{F}=\frac{\delta s}{2 L \underline{\tau}}
$$

It may also be useful to introduce a dimensionless gain of the system as the quotient of the time displacement in one turn due solety to the bump and the instantaneous net time displacement of the reference particle

$$
G=\frac{\delta S}{2 c \tau}=\alpha_{F} T_{0} \text {. }
$$

Another important quantity is the maximum value of ss required of the bump to deal with a coherent oscillation of amplitude $\underline{I}_{\max }$.

$$
\delta s_{\max }=2 L a_{F} I_{\max } .
$$

In PEP we may typically be dealing with instabilities having antidamping coefficients of the order of $1000 \mathrm{sec}^{-1}$ according to Reference 2 , and $T_{0}=7.3 \times 10^{-6} \mathrm{sec}$. To control such a growth rate in a PEP-11ke model ring, $\alpha_{F}$ t $1000 \mathrm{sec}^{-1}$ and

$$
6 \approx 1.4 \times 10^{-2} \text {. }
$$

The rms bunch length will be of the order of $2 \mathrm{~cm}$ which corresponds to an rims time width $\sigma_{t}=67 \times 10^{-12} \mathrm{sec}$. On the basis of SPEAR experience I choose as the maximum coherent amplitude with wich the feedback system has to deal 


$$
\underline{I}_{\max }=3 \sigma_{t}=0.2 \times 10^{-9} \mathrm{sec} .
$$

According to Eq. (15) then the path-length increment required of the bump will be

$$
\delta s_{\max }=0.88 \times 10^{-3} \mathrm{~m}
$$

$L$ being taken to be $2200 \mathrm{~m}$.

To sumarize our results thus far, we have found that in a PEP-size model storage ring with a single PEP-size bunch, we can introduce damping of coherent ?ongitudinal bunch motion through path-length modulation by means of an idea? beam bump. To achieve a damping time of $1 \mathrm{~m} i 11$ isecond we need a gain parameter as defined in Eq. (4) of $1.4 \times 10^{-2}$, and to handle oscillations of time amplitude as large as $0.2 \times 10^{-9} \mathrm{sec}$, which correspond to phase oscillations of amplitude 25 degrees at the PEP radiofrequency of $353 \mathrm{MHz}$. the bump must produce a path-length variation of the reference orbit of 0.88 mill imeter.

\section{The Beam-Bump}

What sort of a beam bump do we need to accomplish this? The form of the radial excursion within the bump is

$$
x_{b}(s)=\theta B_{0}^{\frac{1}{2}} B^{\frac{1}{2}}(s) \sin \phi(s) \text {. }
$$

where $\theta$ is the angular deflection produced by the initial kicker where the betatron function is $\beta_{0}$. This excursion creates a variation to the first order in $x_{b}$ in the circumference of the reference orbit given by

$$
\delta s=\int_{b u \text { upp }} \frac{x_{b}(s)}{s(s)} d s .
$$


A beam bump in a straight section where the bending radius $p$ is infinite does us no good, so we must contrive to produce the bump in a curved section packed with bending magnets. Assuming we can do that,

$$
\delta s=\theta \beta_{0}^{\frac{1}{2}} \int_{\text {bump }} \frac{\beta^{\frac{2}{2}}(s)}{\rho(s)} \sin \phi(s) d s .
$$

For estimating purposes we shall take the betatron function as constant at a typical value $\bar{\beta}$ in the bump and noting that ds = Bd $\phi$ write our estimate

$$
\delta s=\frac{\theta \bar{B}^{2}}{\bar{R}} \int_{0}^{\pi} \sin \phi d \phi
$$

where $\bar{k}$ is a sort of mean local bending radius to take account of the fact that the spaces between bending magnets do not contribute to the integra1. Our estimate then is

$$
\delta s=\frac{2 \theta \bar{B}^{2}}{\bar{R}} \text {. }
$$

so

$$
\theta_{\max }=\frac{\bar{R} \delta s_{\max }}{2 \bar{B}^{2}}
$$

specifies the maximum deflection angle the kicker needs to produce. Note also that in these approximations the amplitude of the beam bump is

$$
a_{b}=\theta \bar{\beta}=\frac{\bar{R} \delta s_{\max }}{2 \bar{\beta}} .
$$


For our PEP-like ring I shall use

$$
\begin{aligned}
& \bar{B}=15 \mathrm{~m}, \\
& \bar{R}=240 \mathrm{~m} .
\end{aligned}
$$

for which

$$
\begin{aligned}
\theta_{\max } & =0.47 \times 10^{-3} \\
a_{b \max } & =7 \times 10^{-3} \mathrm{~m} .
\end{aligned}
$$

Looking at the last figure first, the amplitude of the bump is small compared to the horizontal aperture, 50 the feedback system does not require much extra aperture. Of course, if the damping is successful such large amplitudes, should they be created, would last for only a fraction of a millisecond. The maximum angular kick required $\theta_{\max }$ : while not trivial, does not seen formidable, especially in view of the fact that the most pernicious instability growth rates are expected at the lowest beam energies where the beam is easiest to bend. For example, at $5 \mathrm{GeV}$ an angular kick of 0.47 milliradian is produced by a magnetic field-length product of 80 gauss-meters.

\section{Remarks}

I conclude that the feedback systen described here is probably feasible in an idealized PEP-Tike storage ring; however, it would be remiss not to acknowledge several difficultles in realizing a system based on the idea presented here in an actual storage ring. For one thing, it will be hard to find space along the orbit for the kickers in the arcs which tend to be crowded with equipment already and even harder to find two spaces near enough one half a betatron waveiength apart. Moreover, if the horizontal phase-shift-per-cell is varied, a fixed pair of kickers does not remain at the proper separation. These difficulties might be ameliorated though by the use of a single controlled kicker which excites damped betatren oscillations the sum of which produce, on any turn, the desired path-]ength variation. 


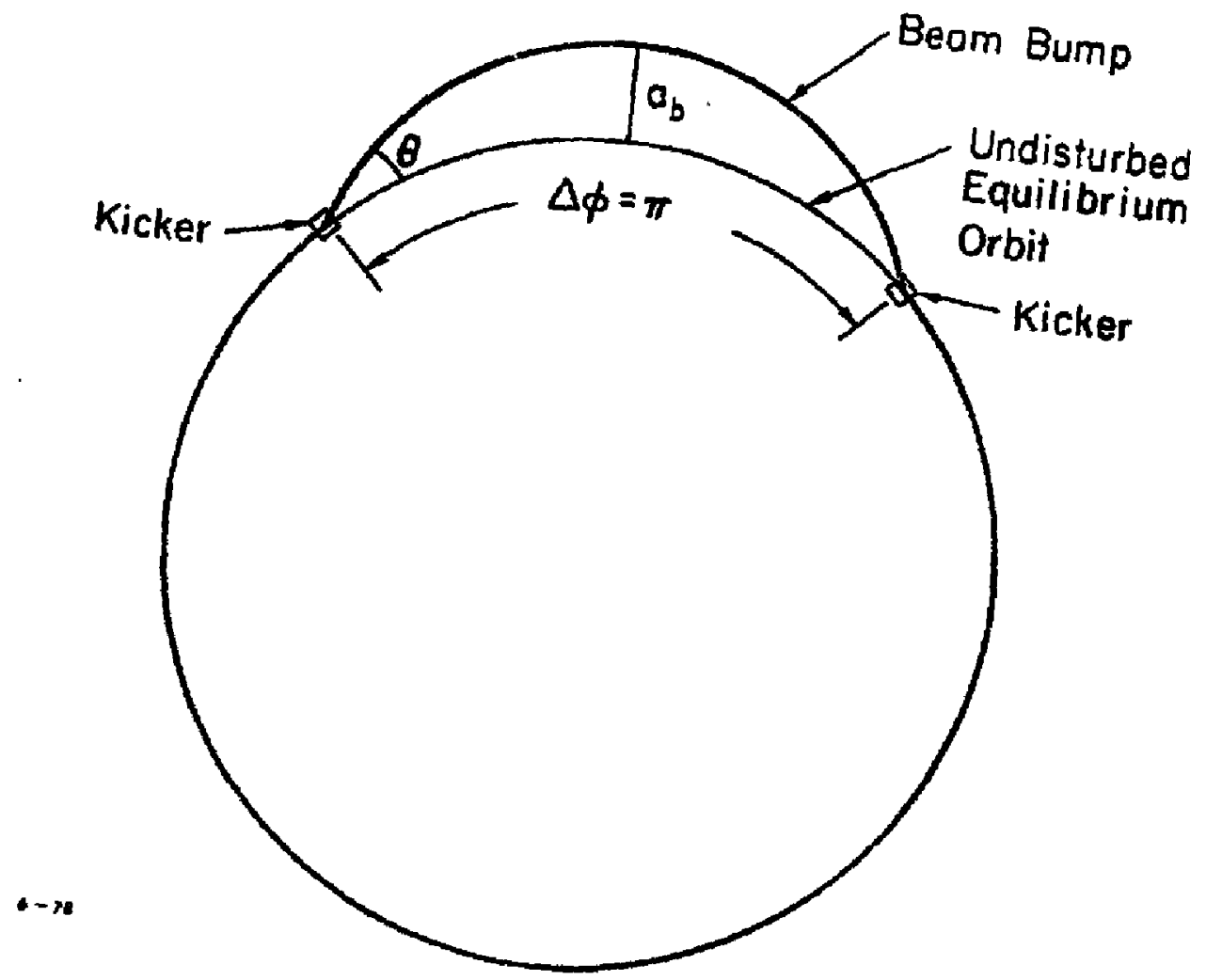

202041

Fig. 1 
Another difficulty - or more precisely a complication - is the application of the method to a multi-bunch beam with its severat nomal modes or coherent rigid-bunch oscillations. And I am sure there are other difficulties. Nevertheless, I believe the method of path-length modulation has promise and deserves further investigation.

\title{
Acknowledgements
}

It is a pleasure to acknowledge valuable and stimulating discussions with Alexander Chao, Phil Morton, Ewan Paterson and Helmut Wiedemann.

\section{References}

1. M. Sands, "The Physics of Electron Storage Rings - An Introduction" in Proceedings of the International School of Physics "Enrico Femi". Varemna, June 1969 (Academic Press, New York).

2. C. Peliegrini and M. Sands, PEP-258, October 1977.

\section{DISCLAIMER}

\begin{abstract}
This report wes prepared as an account of wark sponsored by an agency of the United States Govemmen. Neither the United Sintes Gover nerent nor any agency thertor, nor any of their employees, makes any warranty, express or implied, or assumes any legal liability of responsibility for the accuracy, compteteness, or usefulnese of any informetion, apparalus, product, or process disclosed, or sepresents that its use would not infringe privalely owned rights. Refer: ence hereia to any specific commercial product, prosess, or service by trade name, trademark. monutacturer, of olherwite does not necessarily constitule or imply its endersetreti, recounmendation, of lavoring by the United States Goveroment of any agency thereot. The views and opinions of authors expressed heztin do nok necessarily state or reflect those of the United States Coverninent or ady agency lhetcol.
\end{abstract}

